\title{
Sub-block Aided OFDM with Index Modulation
}

\author{
Y. ACAR
}

\begin{abstract}
Recently, orthogonal frequency division multiplexing (OFDM) with index modulation (IM) has been appeared as a novel method for future wireless communication systems. However, such a mechanism has low spectral efficiency since some sub-carriers are not activated in order to implicitly convey information. In this paper, a subblock dependent approach, called sub-block aided OFDMIM (SA-OFDM-IM) technique, is proposed for spectral efficiency enhancement of the OFDM-IM method with lower complexity. The simulation results illustrate that the proposed SA-OFDM-IM and well known OFDM-IM have same bit error rate (BER) performance while SA-OFDMIM has $40 \%$ more spectral efficiency with low complexity.
\end{abstract}

Index Terms - Orthogonal frequency division multiplexing (OFDM), index modulation (IM), spatial modulation (SM).

\section{INTRODUCTION}

$M$ ULTIPLE-ANTENNA techniques constitute a key technology for next-generation fixed and mobile wireless communication systems [1]. Their performance is related to some restriction such as the spacing between transmitter and receiver antennas [2], [3], inter-antenna synchronization (IAS) at the transmitter and also inter-channel interference (ICI) [4], [5]. To circumvent these problems, Mesleh et al. proposed spatial modulation (SM) as a low computational complexity alternative to well known MIMO systems [6]. The SM technique is using the indices of transmit antennas to carry extra data, in addition to the 2 dimensional $M$-ary signal constellations. To show the potential benefits of $\mathrm{SM}$, several experimental studies have done and it has shown that SM scheme is an alternative to the plain MIMO systems [7]. Consequently, the SM is promising transmission technique that has a very flexible structure and provides low complexity with high spectral efficiency [8].

Orthogonal frequency division multiplexing (OFDM) is being used by wireless communications systems such as the world wide interoperability for microwave access (WiMAX), long term evolution (LTE), IEEE 802.11 (Wi-Fi), etc. [9], [10], [11], [12]. As, OFDM has become the very popular multi-carrier modulation method, it continues to be widely studied. Following this trend, the index modulation (IM)

Yusuf ACAR, is with Department of Electrical and Electronic Engineering, Istanbul Kültür University, Bakırköy, Istanbul, 34156, Turkey, (e-mail: y.acar@iku.edu.tr).

(iD https://orcid.org/0000-0002-3956-1097

Manuscript received January 17, 2019; accepted March 26, 2019.

DOI: $\underline{10.17694 / \text { bajece. } 514324}$ concept which is inspired by SM has draw attention as a promising technique for the new-generation wireless communication systems in the last decade [13]. It has many significant advantages such as reduced peak-to-average power ratio (PAPR), better bit-error-rate (BER), energy efficiency, higher robustness against the inter-carrier interference (ICI) etc. [13], [14], [15].

In [16] and [17] MIMO-OFDM-IM technique is proposed by combining MIMO and OFDM-IM transmission methods. In [18] sub-carrier block interleaving is proposed for OFDMIM to enhance its error performance. The bit error probability of OFDM-IM is analytically derived in [19]. Recently, generalization of OFDM-IM has been proposed by extending the index domain to include quadrature and inphase dimensions [20]. For more details on these studies, the concerned readers are referred to [21].

In spite of its advantages mentioned above, there is still limitation to the operation of OFDM-IM in wireless communications. The OFDM-IM scheme cause a decrease in data rate due to its unused subcarriers. To solve this difficulty, this paper proposes a new OFDM-IM with improved spectral efficiency and low complexity through integrating a new constellation design using sub-block. It is also shown that proposed SA-OFDM-IM performs same performance with traditional OFDM while SA-OFDM-IM has more spectral efficiency with low complexity.

The remain of this study is given as; Section II demonstrates the principle of proposed SA-OFDMIM scheme. Section III introduces the receiver part of the SA-OFDM-IM. The computational complexity and simulation results are given in Section IV and Section V, respectively. Finally, conclusion is presents in Section VI.

\section{PROPOSED SA-OFDM-IM METHOD}

In [13], sub-carriers were partitioned into $g$ subblocks which have $n$ sub-carriers. In each sub-blocks only $k$ out of these $n$ sub-carriers are active, and the remains are inactive. The total number of transmitted data bits for OFDM-IM system is calculated as

$$
m_{I M}=\left\lfloor\log _{2}(C(n, k))\right\rfloor g+k \log _{2}(M) g .
$$




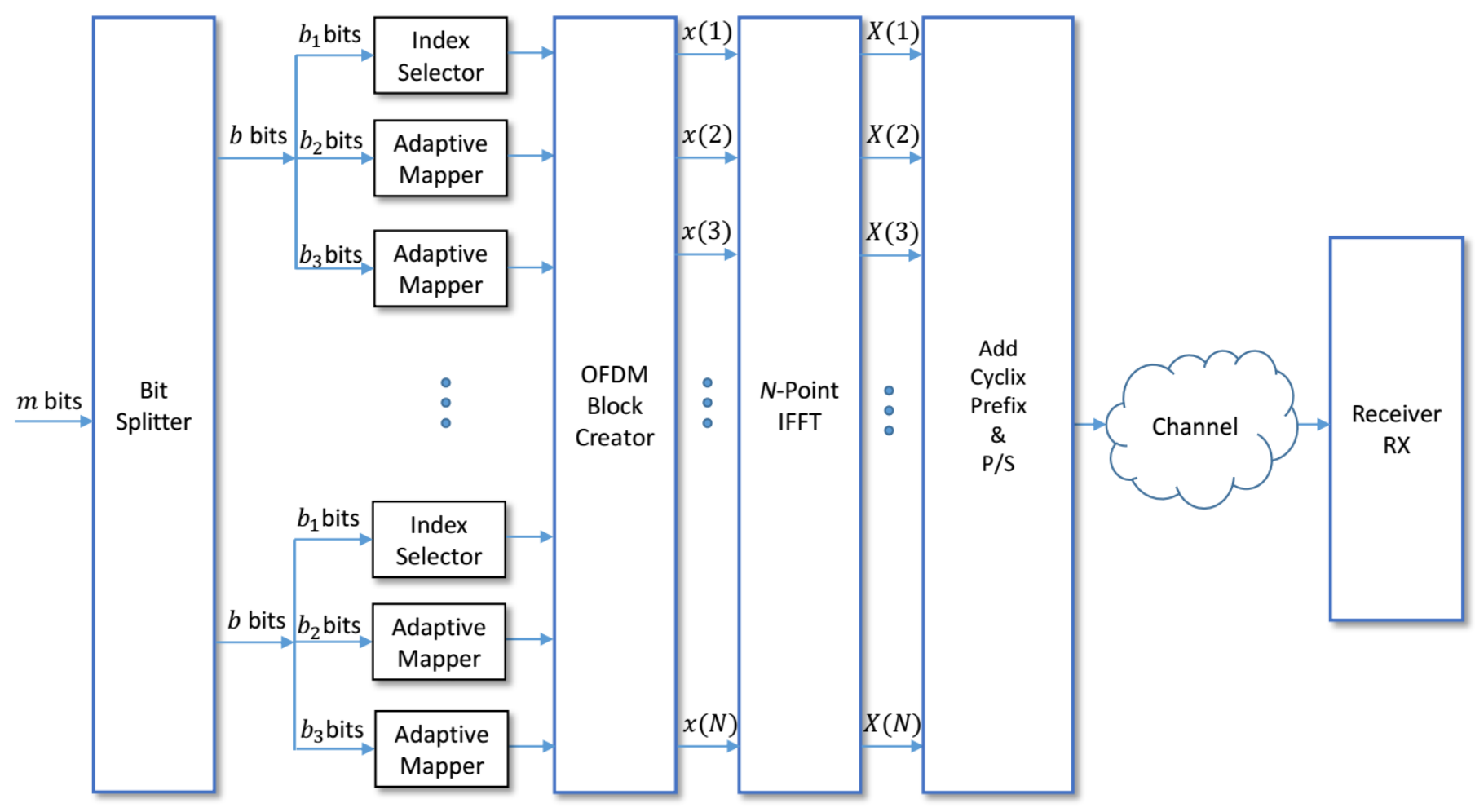

A LOOK-UP TABLE FOR SYSMBOL PAIRS GIVE IN FIG.1 (b)

Fig.1. Block diagram of the proposed scheme

where $M$ represents the modulation size, $\lfloor$.$\rfloor is the floor$ operation and $C(n, k)$ shows the binomial number. It is clear that, unused sub-carriers cause the low spectral efficiency. In this work, sub-block aided OFDM-IM (SA-OFDM-IM) is proposed, to gain a high spectral efficiency rate without degrading BER performance and maintaining same power consumption.

Block diagram of the proposed scheme is given in Fig. 1. To take advantage of sub-blocks (not subcarriers), we design the new constellation scheme as shown in Fig. 2. We split all symbols in a pairs. For example, in Fig. $2(b)$, there are two shifted binary phase shift keying (BPSK) symbol pairs $\left(\left\{x_{1}^{1}, x_{1}^{2}\right\},\left\{x_{2}^{1}, x_{2}^{2}\right\}\right) \in S_{a}$ hence there are two BPSK symbol pairs i.e. $\mathrm{M}=2$. Similarly, symbol pairs in Fig. 2 (c) are given as $\left(\left\{x_{1}^{1}, x_{1}^{2}\right\},\left\{x_{2}^{1}, x_{2}^{2}\right\},\left\{x_{3}^{1}, x_{3}^{2}\right\},\left\{x_{4}^{1}, x_{4}^{2}\right\}\right) \in S_{b}$, thus $\mathrm{M}$ is 4 .

TABLE I

A LOOK-UP TABLE FOR SYSMBOL PAIRS GIVE IN FIG.1 (a)

\begin{tabular}{cc}
\hline Bits & Symbol Pairs \\
\hline$[0]$ & $\left\{x_{1}^{1}, x_{1}^{2}\right\}$ \\
{$[1]$} & $\left\{x_{2}^{1}, x_{2}^{2}\right\}$ \\
\hline
\end{tabular}

We simply assign the information bits in each symbol pairs as given in Tables I-II for proposed constellation diagram. Then, for each sub-block, we used these symbol pairs according to incoming information bits. For instance, if

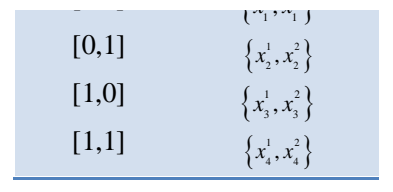

incoming bit for symbol pairs is [0], we use symbol pair $\left\{x_{1}^{1}, x_{1}^{2}\right\}$ in the related sub-block as given in Table I. Similarly, if incoming bits for symbol pairs are [1 1 ], we use symbol pair $\left\{x_{4}^{1}, x_{4}^{2}\right\}$ in the related sub-block as given in Table II. Moreover, each symbol has one information bit such as $x_{2}^{1} \rightarrow[1]$ and $x_{2}^{2} \rightarrow[0]$ as shown in Fig. 2. These designs also can be extend for $M$-ary signal constellation.

\begin{tabular}{|c|c|c|}
\hline Bits & Sub-block IfdeiBdsE III & Sub-block Symbols \\
\hline$A \operatorname{LOC}^{-}[0,0]$ & $\{2,3\}$ & {$\left[x_{i}^{j}, x_{i}^{j}, 0,0\right]$} \\
\hline$[0,1]$ & $\{3,2\}$ & {$\left[0, x_{i}^{j}, x_{i}^{j}, 0\right]$} \\
\hline$[1,0]$ & $\{4,3\}$ & {$\left[0,0, x_{i}^{j}, x_{i}^{j}\right]$} \\
\hline$[1,1]$ & $\{4,1\}$ & {$\left[x_{i}^{j}, 0,0, x_{i}^{j}\right]$} \\
\hline
\end{tabular}

TABLE II

A LOOK-UP TABLE FOR SYSMBOL PAIRS GIVE IN FIG.1 (b) 


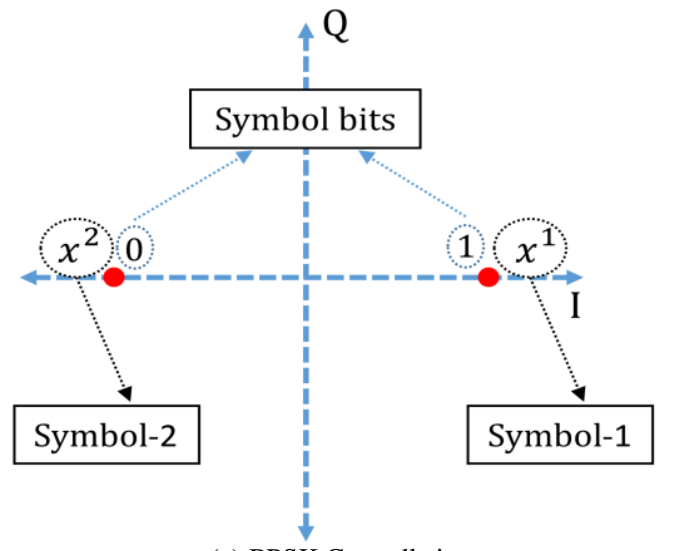

(a) BPSK Constellation

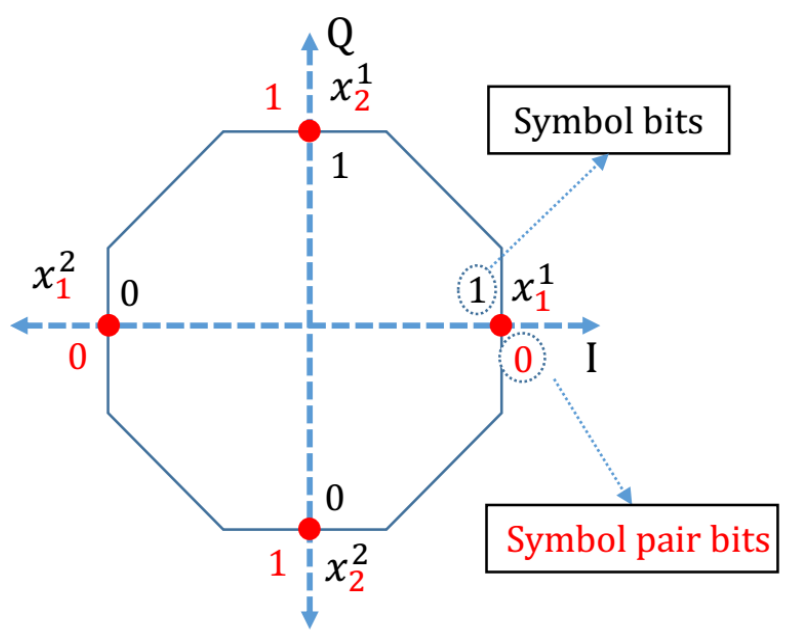

(b) $\mathrm{M}=2$

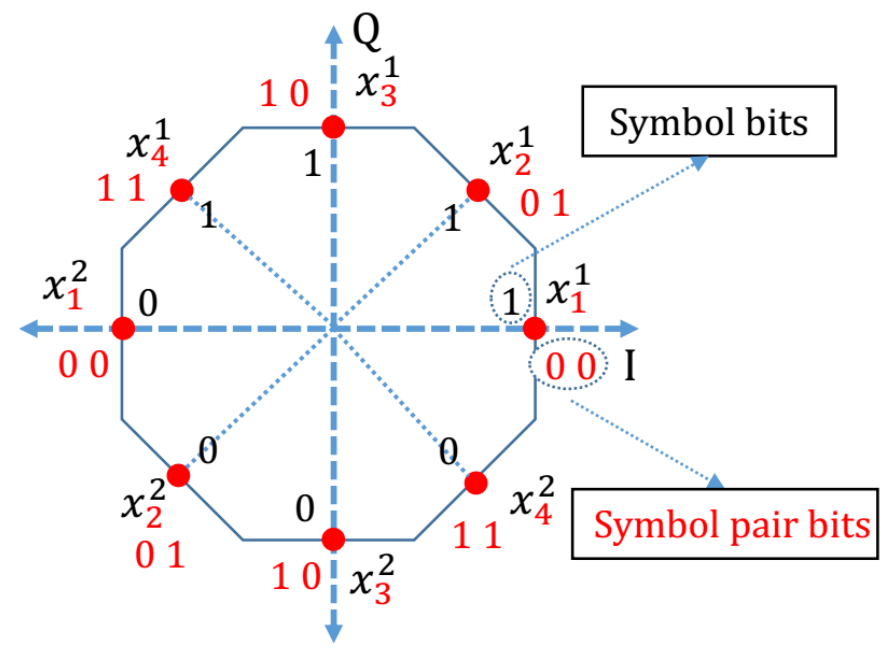

(c) $\mathrm{M}=4$

Fig.2. Proposed constellation diagram for total number of symbol pairs for (b) $M=2$ and (c) $M=4$
The total transmitted bits of proposed scheme can be determined as

$$
\begin{aligned}
m_{S A} & =\left\lfloor\log _{2}(C(n, k))\right\rfloor g+k \log _{2}(M) g+\log _{2}(\mathrm{M}) g \\
& =m_{I M}+\log _{2}(\mathrm{M}) g .
\end{aligned}
$$

Therefore, in the proposed system, in contrast to OFDMIM, all sub-block within the whole OFDM symbol are used to carry additional bits. If the SAOFDM-IM and existing OFDMIM techniques are compared, the total number of data bits, i.e. spectral efficiency of the SA-OFDM-IM technique is greater than that of the existing OFDM-IM schemes. For example, OFDM-IM has $m_{I M}=256$ bits for BPSK modulation while SA-OFDM-IM has $m_{S A}=320$ bits for $\mathrm{M}=2$. Using sub-block also increases the frequency diversity gain in addition to the diversity gain brought by IM.

In the proposed system, incoming bits are divided into $b=m_{S A} / g$ bits. Hence, each sub-block contains $b$ bits. In the each sub-block, the $b$ bits are split into three parts as $b=b_{1}+b_{2}+b_{3}$ for different goals. The first part, $b_{1}$ bits, is used to determine the sub-carrier index as given in Table III. The second part, $b_{2}$ bits, is used by symbol pairs as given Tables I-II. Finally, $b_{3}$ bits is used by modulated symbol $x_{i}^{j}, j=1,2, i=1, \ldots, \mathrm{M}$.

Fig. 3 shows an illustrative frame structure of the proposed scheme where total number of sub-carrier $N=16, n=4$ and $k=2$. We give two example below for better understating.

\section{Example 1:}

Assume that incoming bits are $\left[\begin{array}{llllll}0 & 1 & 0 & 0 & 1 & 0\end{array}\right]$ for first subblock as shown in Fig. 3. The first two bits [0 1] show the subcarriers index, (i.e., in transmission second and third subcarriers will be activated) then other two bits [0 0$]$ are denote the symbol pair index $\left\{x_{i}^{1}, x_{i}^{2}\right\}, i=1, \ldots, \mathrm{M}$ where $\mathrm{M}=4$ and $i=1$ as given in Table II. The last two bits [1 0 ] are for the symbol bits of $x_{1}^{1} \rightarrow[1]$ and $x_{1}^{2} \rightarrow[0]$.

\section{Example 2:}

In this example, incoming bits are $\left[\begin{array}{llllll}1 & 0 & 1 & 0 & 0 & 1\end{array}\right]$ for fourth sub-block. The first two bits [1 0$]$ illustrate that in transmission fourth and third sub-carriers will be activated. Other two bits [1 0 ] are assigned the symbol pair index $\left\{x_{i}^{1}, x_{i}^{2}\right\}, i=1, \ldots, \mathrm{M}$ where $\mathrm{M}=4$ and $i=3$. Finally, last two bits [0 1] are for the symbol bits of $x_{3}^{1} \rightarrow[1]$ and $x_{3}^{2} \rightarrow[0]$. 


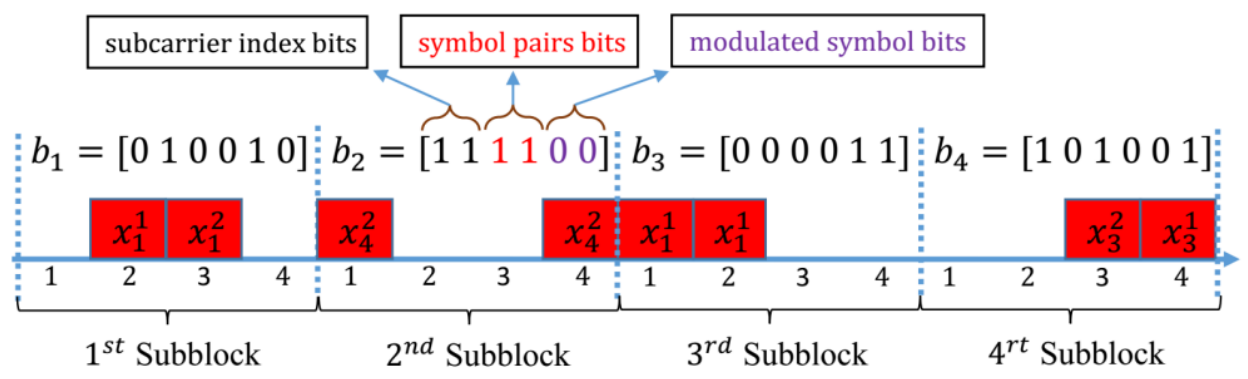

Fig.3. An illustrative frame structure of the proposed scheme with $N=16, n=4$ and $k=2$

By concatenating these $g$ sub-blocks, $\mathbf{x}_{F}$ can be transmitted symbols of the SA-OFDM-IM method:

$$
\begin{aligned}
\mathbf{x}_{F} & =\left[x_{i}^{j}(1), x_{i}^{j}(2), \cdots, x_{i}^{j}(N)\right], \\
j & =1,2, \quad i=1, \ldots, \mathrm{M}
\end{aligned}
$$

Then, after applying IFFT, the OFDM symbol given as:

$$
\mathbf{x}_{T}=\frac{N}{\sqrt{Z}} \mathbf{W}_{N}^{H} \mathbf{x}_{F}
$$

where $\mathbf{W}_{N}$ is the FFT matrix with $\mathbf{W}_{N}^{H} \mathbf{W}_{N}=N \mathbf{I}_{N}, Z=k g$ is the number of active sub-carriers, and $N / \sqrt{Z}$ term is used for the normalization $E\left\{x_{T}^{H} x_{T}\right\}=N$.

At the receiver, the received signal is given as

$$
y_{F}(f)=h_{F}(f) x(f)+w_{F}(f)
$$

where $w_{F}(f)$ and $h_{F}(f)$ are the noise samples and the channel fading coefficients in the frequency domain, respectively.

\section{MAXIMUM LIKELIHOOD (ML) DETECTOR}

We investigate ML detector to estimate the information from the received symbols for proposed scheme. Owing to channel impulse response knowledge, in order to estimate the modulated symbols $\left(\hat{x}_{i, \beta}^{j}\right)$ and the active indices $\left(\hat{I}_{\beta}\right)$ in each sub-block. Then, estimation of the data symbols and active indices are calculated as follows:

$$
\begin{aligned}
\left(\hat{I}_{\beta}, \hat{x}_{i, \beta}^{j}\right) & =\arg \min _{I_{\beta}, x_{i, \beta}^{j}} \sum_{\gamma=1}^{k}\left|y_{F}^{\beta}\left(i_{\beta, \gamma}\right)-h_{F}^{\beta}\left(i_{\beta, \gamma}\right) x_{i, \beta}^{j}(\gamma)\right|^{2} \\
j & =1,2, \quad i=1, \ldots, \mathrm{M}
\end{aligned}
$$

where $y_{F}^{\beta}\left(i_{\beta, \gamma}\right), i_{\beta, \gamma}$, and $h_{F}^{\beta}\left(i_{\beta, \gamma}\right)$ show the received symbols, the indices corresponding to active indices set of $I_{\beta}=\left\{i_{\beta, 1}, \cdots, i_{\beta, k}\right\}$, and the channel coefficients for the sub- lock $\beta$, respectively. $x_{i, \beta}^{j}, \quad j=1,2, i=1, \ldots, \mathrm{M}$ denotes the vector of modulated signals from designed two dimensional constellation alphabets in Fig. 2. To obtain the transmitted information bits on each sub-block, receiver uses these two estimates, $\hat{x}_{i, \beta}^{j}$ and $\hat{I}_{\beta}$, in the Tables I - II by an inverse mapping process.

\section{COMPUTATIONAL COMPLEXITY (CC) COMPARISON}

The CC of the ML detector for exiting OFDMIM is $\sim \mathrm{O}\left(r M^{k}\right)$ where $r$ denotes the number of possible realizations of the selected active indices and $M$ is modulation order. It is obvious that, the CC of exiting OFDM-IM with BPSK is $\sim \mathrm{O}\left(r 2^{k}\right)$. As seen in Fig. 2, the proposed method uses BPSK and $\pi / 2$ rotated form of BPSK. Hence, the CC of proposed method for the ML detector with $\mathrm{M}=2$ is $\sim \mathrm{O}\left(2 r 2^{k}\right)$. Consequently, the CC of the SAOFDM-IM is $\sim \mathrm{O}\left(\mathrm{Mr} 2^{k}\right)$ while OFDM-IM is $\sim \mathrm{O}\left(r M^{k}\right)$. It is clear that the $\mathrm{CC}$ of our proposed scheme is much lower than OFDM-IM scheme with the higher modulation size.

\section{Simulation Results}

The BER performance of the proposed technique is evaluated for new constellation design with $M=2$ and $M=4$ by assuming frequency selective Rayleigh channels with length $N_{L}$. The total number of sub-carriers in one block is set to $n=4$. The SNR is described as $E_{b} / N_{0}$ where $N_{0}$ is the noise power and $E_{b}$ is energy per bit.

The BERs of OFDM-IM and proposed system are given in Figs. 4-5 as functions of the SNR for $N=256$ and $N=1024$, respectively. As shown in from Fig. 4, the proposed method with $\mathrm{M}=2$ achieves almost same performance of the well known OFDM-IM with BPSK and $N=256$. In that case, the total number of data bits transmitted by the proposed method is $m_{S A}=320$ while OFDM-IM is $m_{I M}=256$ bits. Moreover, proposed method with $\mathrm{M}=2$ and OFDM-IM with QPSK modulation scheme have same number of bits as $m_{S A}=m_{I M}=384$ while proposed method has better 
performance. We also use log likelihood receiver (LLR) for OFDM-IM systems for QPSK modulation to compare with the proposed scheme.

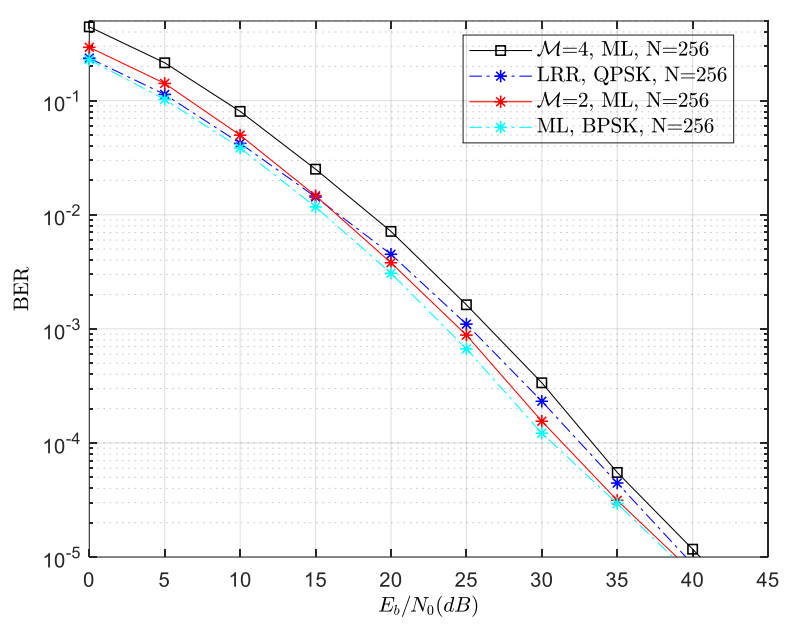

Fig.4. BER performances of proposed SA-OFDMIM scheme and exiting OFDM-IM with $N=256, k=2$ and $n=4$

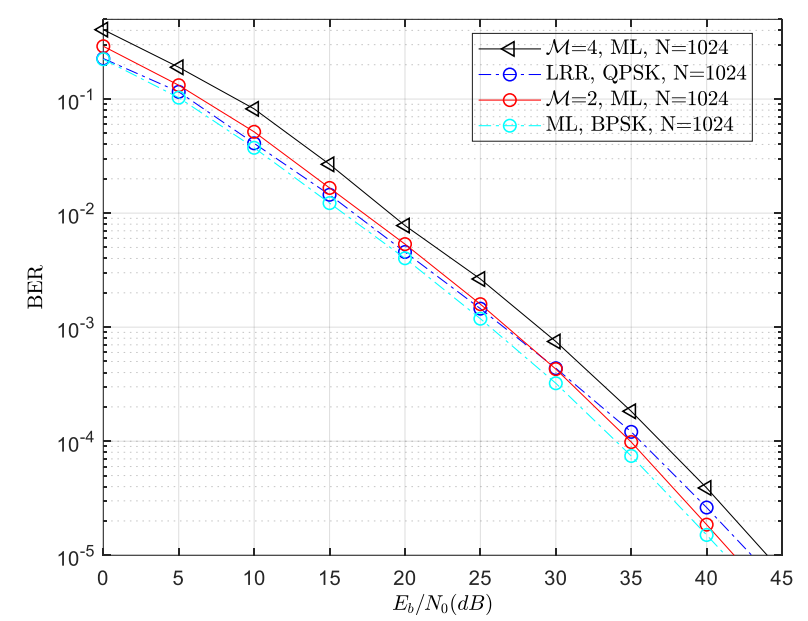

Fig.5. BER performances of proposed SA-OFDMIM scheme and exiting OFDM-IM with $N=1024, k=2$ and $n=4$

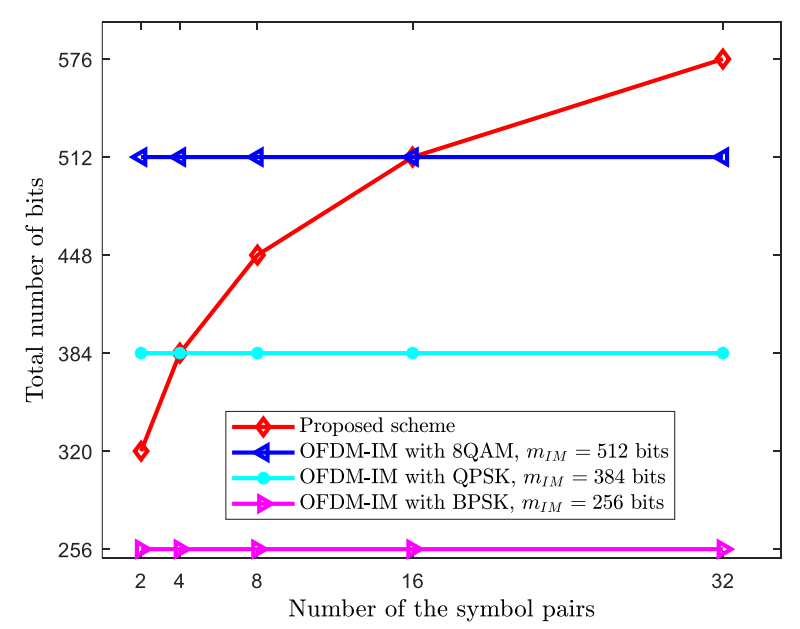

Fig.6. Total number of bits with $N=256, n=4$ and $k=2$

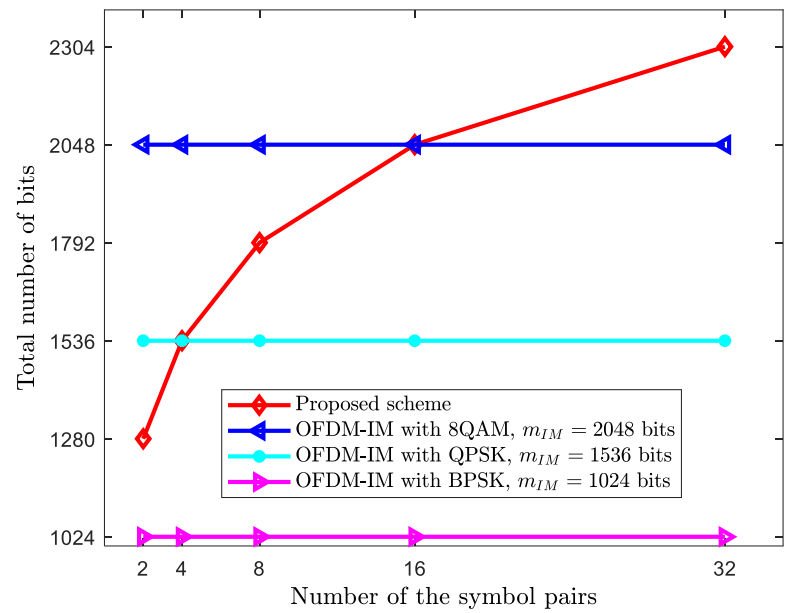

Fig.7. Total number of bits with $N=1024, n=4$ and $k=2$

Figs. 6-7 depicts the impact of increasing the number of the BPSK symbol pairs $M$ on the spectral efficiency of the proposed technique for $N=256$ and $N=1024$, respectively. The spectral efficiency of the proposed method has a high positive correlation with the number of symbol pairs $\mathrm{M}$.

As illustrated in Fig. 7, the total number of bits carry by the proposed scheme with $\mathrm{M}=2$ is $m_{S A}=1280$ while OFDM-IM with BPSK is $m_{I M}=1024$ bits. As a result, the spectral efficiency of the proposed method is $40 \%$ higher than OFDMIM system, while both of them has the same performance and complexity.

\section{CONCLUSIONS}

High spectral efficiency is one of the challenge issue in the next generation wireless communication systems such as $5 \mathrm{G}$, $6 \mathrm{G}$ etc. In this work, to obtain the high spectral efficiency of the OFDM-IM method, new constellation diagrams are designed for each sub-blocks. The bit error rate and total number of bits for proposed method are investigated and the computational complexity is given. Results show that using the proposed constellations in each subblock a high spectral efficiency is achieved with same BER performance and tolerable complexity compared to currently known OFDM IM. In future, this work might be extended to multiple-input multiple-output (MIMO) scheme to increase spectral efficiency more. 


\section{REFERENCES}

[1] N. Panwar, S. Sharma, and A. K. Singh, "A survey on 5g: The next generation of mobile communication," Physical Communication, vol. 18, 2016, pp. 64-84.

[2] A. Sibille, C. Oestges, and A. Zanella, MIMO: from theory to implementation, Academic Press, 2010.

[3] C. Oestges and B. Clerckx, MIMO wireless communications: From realworld propagation to space-time code design, Academic Press, 2010.

[4] S. Ahmadi, LTE-advanced: A practical systems approach to understanding 3GPP LTE releases 10 and 11 radio access technologies. Academic Press, 2013.

[5] A. V. Nikitin, "On the interchannel interference in digital communication systems, its impulsive nature, and its mitigation," EURASIP Journal on Advances in Signal Processing, Vol.2011, No.1, 2011, pp. 137.

[6] R. Mesleh, H. Haas, C. W. Ahn, and S. Yun, "Spatial modulation - A new low complexity spectral efficiency enhancing technique," in Proc. Conf. Commun. Netw. China, Beijing, China, Oct. 2006, pp. 1-5.

[7] N. Serafimovski, A. Younis, R. Mesleh, P. Chambers, M. Di Renzo, C.-X. Wang, P. M. Grant, M. A. Beach, and H. Haas, "Practical implementation of spatial modulation," IEEE Trans. Veh. Technol., Vol.62, No.9, 2013, pp. 4511-4523.

[8] M. Di Renzo, H. Haas, and P. M. Grant, "Spatial modulation for multipleantenna wireless systems: a survey," IEEE Commun. Mag., Vol.49, No.12, pp. 182-191, December 2011.

[9] J. G. Andrews, A. Ghosh, and R. Muhamed, Fundamentals of WiMAX: understanding broadband wireless networking, Pearson Education, 2007.

[10] S. Sesia, M. Baker, and I. Toufik, LTE-the UMTS long term evolution: from theory to practice, John Wiley \& Sons, 2011.

[11] E. Dahlman, S. Parkvall, and J. Skold, 4G: LTE/LTE-advanced for mobile broadband. Academic press, 2013.

[12] B. P. Crow, I. Widjaja, J. G. Kim, and P. T. Sakai, "IEEE 802.11 wireless local area networks," IEEE Communications magazine, Vol.35, No.9, 1997, pp. 116-126.

[13] E. Başar, Ü. Aygölü, E. Panayırcı, and H. V. Poor, "Orthogonal * frequency division multiplexing with index modulation," IEEE Transactions on Signal Processing, Vol.61, No.22, 2013, pp. 5536-5549.

[14] M. Wen, B. Ye, E. Basar, Q. Li, and F. Ji, "Enhanced orthogonal frequency division multiplexing with index modulation," IEEE Trans. Wireless Commun., Vol.16, No.7, 2017, pp. 4786-4801.

[15] N. Ishikawa, S. Sugiura, and L. Hanzo, "Subcarrier-index modulation aided ofdm-will it work?" IEEE Access, Vol.4, 2016, pp. 2580-2593.

[16] E. Başar, "Multiple-input multiple-output OFDM with index modulation," IEEE Signal Processing Letters, Vol.22, No.12, 2015, pp. 22592263 ,

[17] B. Zheng, M. Wen, E. Basar, and F. Chen, "Multiple-input multipleoutput OFDM with index modulation: Low-complexity detector design," IEEE Transactions on Signal Processing, Vol.65, No.11, 2017, pp. 27582772.

[18] Y. Xiao, S. Wang, L. Dan, X. Lei, P. Yang, and W. Xiang, "OFDM with interleaved subcarrier-index modulation," IEEE Communications Letters, Vol.18, No. 8, 2014, pp. 1447-1450.

[19] Y. Ko, "A tight upper bound on bit error rate of joint OFDM and multicarrier index keying," IEEE Communications Letters, Vol.18, No.10, 2014, pp. 1763-1766.

[20] R. Fan, Y. J. Yu, and Y. L. Guan, "Generalization of orthogonal frequency division multiplexing with index modulation," IEEE transactions on wireless communications, Vol.14, No.10, pp. 5350-5359, 2015.

[21] E. Başar, M. Wen, R. Mesleh, M. Di Renzo, Y. Xiao, and H. Haas, "Index modulation techniques for next-generation wireless networks," IEEE Access, Vol.5, 2017, pp. 693-746.

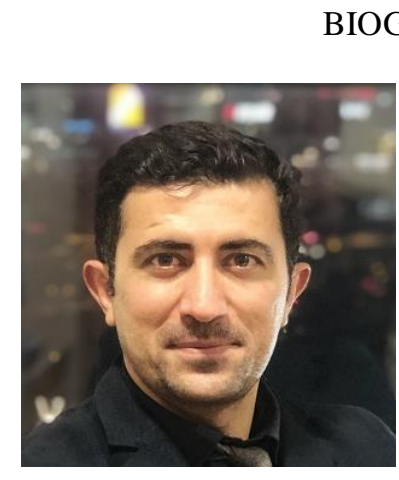

\section{BIOGRAPHY}

YUSUF ACAR received the B.S.E. degree (with honors), M.S.E. degree and Ph.D. degrees in Electrical and Electronics Engineering from Istanbul University, Istanbul, Turkey, in 2008, 2011 and 2015, respectively. In 2015, he joined the Department of Electrical and Electronics Engineering, Istanbul Kültür University as an Assistant Professor.

He was a researcher at the Purdue University Fort Wayne (PFW), USA, between the September 2017 and September 2018. His general research interests cover communication theory, estimation theory, statistical signal processing, and information theory. His current research activities are focused on software-defined platforms. 